TRANSACTIONS OF THE

AMERICAN MATHEMATICAL SOCIETY

Volume 184, October 1973

\title{
FIXED POINT STRUCTURES
}

BY

\section{T. B. MUENZENBERGER( ${ }^{(1)}$ AND R. E. SMITHSON}

ABSTRACT. A fixed point structure is a triple $(X, 9, \mathcal{F})$ where $X$ is a set, 9 a collection of subsets of $X$, and $\boldsymbol{F}$ a family of multifunctions on $X$ into itself together with a set of axioms which insure that each member of $\mathcal{F}$ has a fixed point. A fixed point structure for noncontinuous multifunctions on semitrees is established that encompasses fixed point theorems of Wallace-Ward and Young-Smithson as well as new fixed point theorems for partially ordered sets and closed stars in real vector spaces. Also two other fixed point structures are presented that subsume fixed point theorems of Tarski-Ward-Smithson on semilattices and, more generally, partially ordered sets. Also the Davis-Ward converse to this last fixed point theorem is obtained.

1. Introduction. In 1941 A. D. Wallace [16] proved a fixed point theorem for upper semicontinuous multifunctions on trees. Then Capel and Strother [3] gave a new proof of Wallace's theorem which utilized the inherent partial order structure of trees. In [17] L. E. Ward studied this partial order, and in [20] he used order theoretic techniques to characterize the fixed point property for upper semicontinuous, continuum-valued multifunctions on arcwise connected metric continua. Then in [12] Smithson generalized the fixed point theorem of Ward and a theorem of Young [22] on certain arcwise connected acyclic topological spaces to a class of multifunctions which did not satisfy any of the classical continuity properties. Using the ideas and techniques of the last paper as motivation, a fixed point theory based on a set of axioms is developed in this paper.

The remainder of this section will be devoted to the basic definitions used in the sequel. In $\$ 2$ the axioms on $\mathcal{P}$ will be given and the basic properties of the structure $(X, \mathscr{P})$ developed. In $\$ 3$ the axioms on $\mathcal{F}$ will be given and some properties of the structure $(X, \mathcal{P}, \mathcal{F})$ presented. In $\$ 4$ the main fixed point theorem will be proved, and in $\$ 5$ examples of some fixed point structures which have nearly all of the above results as corollaries will be given. We shall also give an example of a fixed point structure in a topological vector space which has a fixed point

Presented to the Society, January 20, 1972; received by the editors March 3, 1972 and, in revised form, November 22, 1972.

AMS (MOS) subject classifications (1970). Primary 54H25; Secondary 54C60, 54F05.

Key words and phrases. Fixed points, partial order, chain topology, fixed points for a class of multifunctions, arcwise connected spaces, fixed point structures, semitrees.

(l) This work was supported in part by a National Science Foundation Traineeship. 
theorem analogous to the Kakutani theorem [6] as a corollary. Finally, several alternate fixed point structures will be given in $\$ 6$.

A multifunction $F: X \rightarrow Y$ is a point to set correspondence on $X$ into $Y$ with $F(x) \neq \varnothing$ for all $x \in X$, where $\varnothing$ denotes the empty set. If $P$ represents a property, then $F$ is point $P$ in case $F(x)$ has property $P$ for all $x \in X$. Now $F$ is said to be upper semicontinuous (u.s.c.) if and only if $F^{-1}(A)=\{x \in X: F(x) \cap A \neq \varnothing\}$ is closed for each closed $A \subset Y$; also, $F$ is said to be lower semicontinuous (l.s.c.) if and only if $F^{-1}(V)$ is open for each open $V \subset Y$. Further, $F$ is continuum-valued in case $F(x)$ is a continuum for all $x \in X$. A connected multifunction is one which preserves connected sets. A monotone multifunction $F: X \rightarrow Y$ has the property that $F^{-1}(y)$ is connected for each $y \in Y$. Finally, $F: X \rightarrow X$ has a fixed point in case there is an $x \in X$ such that $x \in F(x)$. By a fixed point structure we mean a triple $(X, \mathcal{P}, \mathcal{F})$ where $X$ is a nonempty set, $\mathcal{P}$ is a nonempty collection of subsets of $X$, and $\mathcal{F}$ is a nonempty family of multifunctions on $X$ into $X$ together with a set of axioms on $\mathcal{P}$ and a set of axioms on $\mathcal{F}$ which imply that each $F \in \mathcal{F}$ has a fixed point. For the sake of brevity we shall refer to the triple $(x, \mathcal{P}, \mathcal{F})$ as the fixed point structure. We shall use this notation in succeeding sections.

2. The axioms on $\mathcal{P}$. In this section we present the axioms on $\mathcal{P}$ and derive the basic properties of $(X, \mathcal{P})$ which result from these axioms. After an axiom has been given, it shall be assumed to hold. However, in some cases where only some of the axioms are needed, these will be specified.

Axiom 1. For all $x, y \in X$, there exists a $P \in \mathcal{P}$ such that $x, y \in P$.

Axiom 2. If $\varnothing \neq \mathscr{P}_{0} \subset \mathcal{P}$, then $\cap \mathscr{P}_{0}=\varnothing$ or $\cap \mathscr{P}_{0} \in \mathcal{P}$.

Lemma 2.1. For all $x, y \in X$ there exists a unique minimal (under inclusion) element of $\mathcal{P}$ that contains $x, y$.

Proof. Set $\mathscr{P}_{0}=\{P \in \mathscr{P}: x, y \in P\}$. By Axiom $1, \mathscr{P}_{0}$ is nonempty, and by Axiom 2, $\cap \mathcal{P}_{0} \in \mathcal{P}$. Thus $\cap \mathcal{P}_{0}$ is the required minimal element of $\mathcal{P}$.

The minimal element containing $x, y$ is denoted by $[x, y]$ and is called the chain in $X$ with endpoints $x, y$. We define $(x, y]=[x, y] \backslash\{x\}=[y, x)$ and $(x, y)=$ $[x, y] \backslash\{x, y\}$. Note that $[x, y]=[y, x]$.

Corollary 2.2. The set $X$ does not contain two distinct chains with the same endpoints.

Corollary 2.2 says that $X$ is acyclic or that $X$ contains no circles when Axioms 1 and 2 hold.

Axiom 3. For all $P \in \mathcal{P}$ there exists a unique pair $x, y \in X$ such that $P=[x, y]$.

Axiom 3 says that every element of $\mathcal{P}$ is a chain, and thus $\mathscr{P}$ consists of the chains in $X$. 
Definition. A subset $A \subset X$ is chainable if and only if for all $x, y \in A,[x, y] \subset A$.

The property of being chainable is fundamental to the development of the fixed point structure. Note that $X$ itself is chainable. The proof of Lemma 2.3 is routine and is omitted.

Lemma 2.3. The intersection of chainable sets is chainable.

Lemma 2.4. If $z \in(x, y)$, then $[x, y] \backslash\{z\}$ is not chainable.

Proof. Since $x, y \in[x, y] \backslash\{z\}$ and $[x, y] \not \subset[x, y] \backslash\{z\},[x, y] \backslash\{z\}$ is not chainable.

Remark. Lemma 2.4 is not fundamental to the development of the theory, but it does show that there is a close connection between the notions of chains and arcs. In this context see the remark following Lemma 2.8 .

Axiom 4. The union of two chainable sets with nonempty intersection is chainable.

Axiom 5. If $\mathscr{P}_{0} \subset \mathcal{P}$ is nested, then there exists a $P \in \mathcal{P}$ such that $\cup \mathcal{P}_{0} \subset P$.

Axiom 5 turns out to be of great importance in the development of fixed point structures. It is a form of completeness or compactness, and is an adaptation of the condition used by Young in [22].

Definition. Fix a point $e \in X$ and define a relation $\leq$ by: $x \leq y$ if and only if $x \in[e, y]$. If $x \leq y$ and $x \neq y$, write $x<y$.

Axiom 6. For all $x, y \in X$ with $x<y$, there exists a $z \in X$ such that $x<z<y$.

Axiom 6 could be rephrased in terms of chains as follows: If $x \neq y$, then $[x, y]$ contains at least three points. (See the remark following Lemma 2.19.)

The above six axioms are the axioms that we assume on the pair $(X, \mathcal{P})$. Examples have been found which show that these six axioms are independent. These examples appear in [8].

For the remainder of this section, we shall study the triple $(X, \mathcal{P}, \leq)$ where $\leq$ is the relation defined above with the point $e$ fixed, and we assume that all six axioms hold.

Lemma 2.5. If $x \in X$, then $[x, x]=\{x\}$.

Proof. If $y \in[x, x]$ then by Axiom $2,[x, y] \cap[x, x] \in \mathcal{P}$. Thus by Lemma 2.1, $[x, y]=[x, x]$. Hence, $y=x$ by Axiom 3. Thus $[x, x]=\{x\}$.

Lemma 2.6. If $z \in[x, y]$, then $[x, z] \subset[x, y]$.

Proof. Apply Axiom 2 and Lemma 2.1 .

Lemma 2.7. The relation $\leq$ is a partial order.

Proof. Since $x \in[e, x], \leq$ is reflexive. If $x \leq y$ and $y \leq x$, then $[e, x]=$ $[e, y]$ by Lemma 2.6, and so $x=y$ by Axiom 3. Now let $x \leq y$ and $y \leq z$. Then $x \in[e, y]$, but $[e, y] \subset[e, z]$. So $x \in[e, z]$, and $x \leq z$. 
Note that $e$ is the unique least element for the partial order $\leq$. In the following we shall use the notation $L(x)=\{y \in X: y \leq x\}$ and $M(x)=\{y \in X: x \leq y\}$.

The triple $(X, \mathcal{P}, \leq)$ is called a semitree and has many interesting properties. In addition to the properties of semitrees cited here, a number of other properties will be given in other papers.

If $x \leq y$, we write $[x, y]$ rather than $[y, x]$ and we call $[x, y]$ an ordered chain (see Corollary 2.14). If $x$ and $y$ are not comparable, then $[x, y]$ is called an unordered chain.

Lemma 2.8. For all $x, y \in X,[x, y]$ is chainable.

Proof. This follows from Axiom 2 and Lemma 2.1.

We could also prove that $[x, y),(x, y]$ and $(x, y)$ are all chainable in contrast to Lemma 2.4 .

Corollary 2.9. The following statements bold: (i) For eacb $x \in X, L(x)$ is chainable. (ii) For all $x, y \in X$ with $x \leq y,[x, y] \subset L(y)$. (iii) If $x \leq y$, then $[e, x] \subset[e, y]$.

Proof. Since $L(x)=[e, x]$, statement (i) follows from Lemma 2.8, and (i) implies (ii) and (iii).

Lemma 2.10. If $x \leq y$, then $L(y)=L(x) \cup[x, y]$.

Proof. By Lemma 2.8 and Axiom 4, $L(x) \cup[x, y]$ is chainable. Then, since $e, y \in L(x) \cup[x, y], L(y) \subset L(x) \cup[x, y]$. Further, $x \leq y$ implies $L(x) \subset L(y)$ and $[x, y] \subset L(y)$ by Corollary 2.9. Thus $L(x) \cup[x, y] \subset L(y)$ and the lemma follows.

Corollary 2.11. If $x \leq y$, then $[x, y] \subset M(x)$.

Proof. If $z \in[x, y]$, then $x \in L(z)$ by Lemma 2.10, and so $z \in M(x)$.

Corollary 2.12. If $x \leq y$, then $L(x) \cap[x, y]=\{x\}$ and $L(y) \backslash L(x)=(x, y]$.

Proof. That $L(x) \cap[x, y]=\{x\}$ follows from Corollary 2.11 since $\leq$ is a partial order. The second statement follows from $L(x) \cap M(x)=\{x\}$ and Corollary 2.11.

Lemma 2.13. For each $x \in X, L(x)$ is totally ordered by $\leq$.

Proof. Let $y, y^{\prime} \in L(x)$ with $y \neq y^{\prime}$. By Lemma 2.10, $L(x)=L(y) \cup[y, x]$. Thus $y^{\prime} \in L(y)$ or $y^{\prime} \in[y, x]$. If $y^{\prime} \in L(y), y^{\prime} \leq y$. So assume $y^{\prime} \in[y, x]$. Then by Corollary $2.11,[y, x] \subset M(y)$. Thus $y \leq y^{\prime}$ and the lemma follows.

Corollary 2.14. If $x \leq y$, then $[x, y]$ is totally ordered.

Proof. By Lemma 2.10, $[x, y] \subset L(y)$, and each subset of a totally ordered set is totally ordered.

For $x, y \in X$, we define $x \wedge y=\sup (L(x) \cap L(y))$. It is easy to prove that 
$x \wedge y$ exists for each $x, y \in X$. As a consequence, the pair $(X, \leq)$ is a semilattice.

Lemma 2.15. For each pair $x, y \in X, x \wedge y$ exists.

Proof. Let $x, y \in X$. Then $L(x) \cap L(y) \in \mathcal{P}$ (by Axiom 2), and so $L(x) \cap$ $L(y)=[a, b]$ for a unique pair $a, b$. By Lemma $2.13,[a, b]$ is totally ordered. Thus assume that $a \leq b$. Now $e \in[a, b]$ and $[a, b] \subset M(a)$ by Corollary 2.11. Thus $a=e$. Next note that $L(x) \cap L(y)=[e, b]=L(b)$. Thus $b$ is the supremum of $L(x) \cap L(y)$, and hence, $x \wedge y=b$.

Corollary 2.16. For all $x, y \in X, L(x) \cap L(y)=L(x \wedge y)$.

Remark. While $(X, \leq)$ is a semilattice, it will be a lattice only if it is totally ordered. For if $x, y \in X$ are not comparable, then $x$ and $y$ do not even have an upper bound. (This follows from Lemma 2.13 and the definition of $\leq$.)

Lemma 2.17. If $x \leq y$, then $[x, y]=L(y) \cap M(x)$.

Proof. By Lemma 2.10 and Corollary 2.11, $[x, y] \subset L(y) \cap M(x)$. Now let $z \in L(y) \cap M(x)$. Then $x \leq z \leq y$. If $x=z$ we are done, so suppose that $x<z$. Then, since $L(y)=L(x) \cup[x, y], z \in[x, y]$, and so $L(y) \cap M(x) \subset[x, y]$.

Corollary 2.18. If $x \leq y \leq z$, then $[x, y] \cup[y, z]=[x, z]$.

Proof. Since $x \leq y \leq z$,

$$
\begin{aligned}
{[x, y] \cup[y, z] } & =(M(x) \cap L(y)) \cup(M(y) \cap L(z)) \\
& =(M(x) \cup(M(y) \cap L(z))) \cap(L(y) \cup(M(y) \cap L(z))) \\
& =M(x) \cap L(z)=[x, z] .
\end{aligned}
$$

Lemma 2.19. For all $x, y \in X$, (i) $[x, y]=[x \wedge y, x] \cup[x \wedge y, y]$, (ii) $[x \wedge$ $y, y] \cap[x \wedge y, x]=\{x \wedge y\}$ and (iii) if $x, y$ are not comparable, then no $p \epsilon$ ( $x \wedge y, x]$ is comparable to any $q \in(x \wedge y, y]$.

Proof. First we show (i) holds. By Axiom 4, $[x \wedge y, x] \cup[x \wedge y, y]$ is chainable, and hence, $[x, y] \subset[x \wedge y, x] \cup[x \wedge y, y]$. Next note that if $x \wedge y \in[x, y]$, then both $[x \wedge y, x]$ and $[x \wedge y, y]$ are contained in $[x, y]$, and we are done. Thus there is an $a \in(x \wedge y, x]$ and a $b \in(x \wedge y, y]$ such that $[x \wedge y, x] \cap[x, y]$ $=[a, x],[x \wedge y, y] \cap[x, y]=[b, y]$ and $[a, x] \cup[b, y]=[x, y]$. Then $[a, b] \subset$ $[x, y]$. But $[a, b] \subset[x \wedge y, a] \cup[x \wedge y, b]$ also, and so $[a, b]=\{a, b\}$. Now pick $d$ such that $x \wedge y<d<a$. By an argument analogous to the one just completed, $[d, b]=\{d, b\}$. But now $[a, b] \cup[d, b]$ is chainable and equals $\{a, b, d\}$; thus $[d, a]=\{d, a\}$ which contradicts Axiom 6 since $d<a$, and (i) follows.

Next if $z \in[x \wedge y, x] \cap[x \wedge y, y]$, then $z \in L(x) \cap L(y)$ and thus $z=x \wedge y$, and (ii) follows. Finally, if $p \in(x \wedge y, x]$ is comparable to $q \in(x \wedge y, y)$, then 
either $p$ or $q$ is an element of $[x \wedge y, x] \cap[x \wedge y, y]$ which contradicts (ii). Thus (iii) follows.

Remark. The proof of part (i) of Lemma 2.19 shows that the remark following Axiom 6 gives a statement equivalent to Axiom 6 .

Lemma 2.20. For all $x \in X, M(x)$ is chainable.

Proof. Let $x_{1}, x_{2} \in M(x)$. By Corollary $2.11,\left[x, x_{1}\right]$ and $\left[x, x_{2}\right]$ are contained in $M(x)$, and $\left[x, x_{1}\right] \cup\left[x, x_{2}\right]$ is chainable by Axiom 4. Thus $M(x)$ is chainable.

The next result is not needed explicitly in the proof of the main theorem. However, it does contain some fundamental properties of the partial order and does depend on all six axioms. In particular the significance of Axiom 5 is shown in the role it plays in proving the existence of maximal elements.

Definition. A ray in $X$ with endpoint $e$ is the union of a maximal nest of chains which have $e$ as a common endpoint.

Theorem 2.21. In a semitree $(X, \mathcal{P}, \leq)$ the following bold: (i) Every ray in $X$ with endpoint $e$ is a chain in $X$ with one endpoint $e$. (ii) For each a $\in X$, there exists a maximal element $x_{0} \in X$ such that $a \leq x_{0}$. (iii) An element $x$ of $X$ is maximal if and only if $[e, x]$ is a ray.

Proof. Let $R$ be a ray in $X$ and let $\mathbb{N}$ be the maximal nest of chains such that $R=\bigcup M$. Then by Axiom 5 there exists a chain $[a, b] \subset X$ such that $R \subset$ $[a, b]$. Now, since $e$ is the least element of $R$ and $R$ is totally ordered, we may assume that $a=e$ and thus $[a, b]=L(b)$. Further, if $M \in \mathbb{N}$, then $M \subset L(b)$ and thus $\mathbb{U} \cup\{L(b)\}$ is a nest of chains. Hence, $R=L(b)$, and (i) follows. For (ii), let $\pi$ be a maximal nest of chains with endpoint $e$ which contains $L(a)$. (We use the Hausdorff Maximality Principle to obtain $\pi$.) We let $L(b)$ be the chain obtained in the proof of (i). Then $b$ is the required maximal element. Finally, if $x_{0}$ is a maximal element, $L\left(x_{0}\right)=\bigcup\left\{L(x): x \leq x_{0}\right\}$, and thus is a ray. This combined with the proof of part (ii) implies (iii).

Next we prove two results which will be needed in the sequel.

Lemma 2.22. The pair $(X, \leq)$ is a complete semilattice, and if $\varnothing \neq A \subset[x, y]$, then inf $A \in[x, y]$.

Proof. Let $A$ be a nonempty subset of $X$. Then $\bigcap\{L(a): a \in A\}=L\left(a_{0}\right)$ for some $a_{0} \in X$. (This follows from the fact that $L(x)$ is totally ordered for each $x \in X$ and Axiom 2.) But then $a_{0}=\inf A$. Now assume that $A \subset[x, y]$. Then $x \wedge y \leq \inf A$ and since $[x, y]=[x \wedge y, x] \cup[x \wedge y, y]$, it follows that inf $A \in[x, y]$.

Lemma 2.23. If $\varnothing \neq A \subset X$ is totally ordered, then $A$ bas a supremum in $X$, and if, moreover, $A \subset[x, y]$, then sup $A \in[x, y]$.

Proof. Since $A$ is totally ordered, the collection $\{[e, a]: a \in A\}$ is a nest of 
chains, and so there is a chain $[e, b]$ such that $A \subset \bigcup\{[e, a]: a \in A\} \subset[e, b]$. Now let

$$
B=\{x \in[e, b]: a \in A \text { implies } a \leq x\} \text {. }
$$

Then there is an $x_{0} \in X$ such that $L\left(x_{0}\right)=\bigcap\{[e, x]: x \in B\}$, and $x_{0}=\sup A$. In proving the second assertion of the lemma, we may assume that $x \leq y$. If $A \subset$ $[x, y]$, then $y$ is an upper bound of $A$ and so $x_{0} \leq y$. Further, $A \subset[x, y]$ implies that $x$ is a lower bound of $A$, and so $x \leq x_{0}$. Thus $x_{0} \in[x, y]$.

Corollary 2.24. If $x \leq y$, then $[x, y]$ is a complete lattice (cf. Ward [18, p. 579, Corollary 1.2]).

Next we introduce the notion of a quasi-least upper bound and a notion of closed for a set $A \subset X$.

Definitions. Let $A$ be a nonempty subset of $X$. Then (i) a point $x \in X$ is a quasi-least upper bound of $A$ in $X$ if and only if (a) for all $a \in A, x \nless a$ and (b) for all $y \in X$ such that $y \nless a$ for each $a \in A, y \nless x$. (ii) The set $A$ is closed below if and only if inf $A \in A$. (iii) The set $A$ is closed above if and only if $A$ contains all of its quasi-least upper bounds.

Definition. A set $A \subset X$ is closed if and only if for all $y, z \in X$ with $y \leq z$, $\inf (A \cap[y, z]) \in A$ and $\sup (A \cap[y, z]) \in A$ whenever $A \cap[y, z] \neq \varnothing$.

Remark. The collection $\mathcal{T}_{C}=\{X \backslash A: A \subset X$ is closed $\}$ is a topology on $X$ which we call the chain topology on $X$. This topology has many interesting properties and will be studied in detail in another paper. (See Lemma 2.28 below.) For now we make some elementary observations. In an arc (see \$5), the chain topology coincides with the usual order topology. In general, however, the chain topology is distinct from the topologies usually imposed on partially ordered sets. For example, consider the closed infinite broom $X$ (Example 120 in [14]). We let $\mathcal{P}$ consist of the arcs in $X$ with associated chain topology $\mathcal{T}_{C}, e$ be the origin in $X, \mathcal{T}_{E}$ be the Euclidean subspace topology and $\mathcal{T}_{I}$ be the interval topology of O. Frink [4]. (The topology $\mathfrak{T}_{I}$ is generated by taking all of the sets $L(x)$ and $M(x), x \in X$, as a subbasis for the closed sets.) Then these three topologies are related by inclusion as follows:

$$
\mathfrak{T}_{I} \subsetneq \mathcal{T}_{E} \subsetneq \mathfrak{T}_{C}
$$

(The unit disc in the plane is a semitree with exactly the same properties.)

There are even dendrites (hereditarily unicoherent, locally connected, metric continua) whose topology is strictly contained in the chain topology. To construct a simple example, erect at $1 / 2^{n}$ on $[0,1]$ an interval of length $1 / 2^{n}, n=0,1,2, \ldots$. Let $Y$ be the union of $[0,1]$ and the erected intervals. If we let $\mathcal{P}$ consist of the arcs in $Y$ and let $e=0$, then $Y$ is a semitree which might be called an infinite pinched comb. Letting $\mathfrak{J}_{l}, \mathfrak{J}_{E}$ and $\mathfrak{T}_{C}$ be defined as in our first example, we 
have exactly the same relationship among the topologies as above. However, $Y$ is in several respects a more interesting example than $X$. First, the set $Y$ has the structure of a one dimensional simplicial complex $K$, and the associated Whitebead topology. $\mathcal{T}_{W}$ is even larger than the chain topology:

$$
\mathfrak{T}_{C} \subsetneq \mathfrak{T}_{w^{*}}
$$

(The Whitehead topology is the largest topology which induces the order topology on each simplex in $K_{\text {.) }}$ Second, each of $\mathfrak{T}_{l}, \mathfrak{T}_{E}$ and $\mathfrak{T}_{C}$ admits a particularly nice subbasis for the closed sets. A subset $A \subset Y$ is totally unordered if and only if no two distinct elements of $A$ are related under the semitree ordering $\leq$ with minimal element $e$ induced on $Y$ by $\mathcal{P}$. If $A \subset Y$, then we set $L(A)=\{y \in Y: y \leq a$ for some $a \in A\}$ and $M(A)=\{y \in Y: a \leq y$ for some $a \in A\}$. If $\mathbb{Q}$ is a family of subsets of $Y$, then $\mathcal{T}(\mathbb{Q})$ denotes the topology with subbasis for the closed sets consisting of $L(A)$ and $M(A), A \in \mathbb{Q}$. We now let $\mathbb{Q}_{0}$ consist of the singletons in

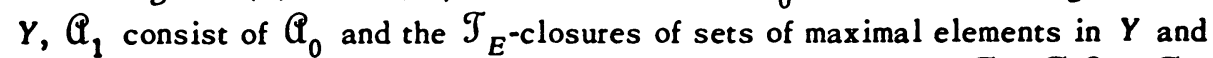
$\mathbb{Q}_{2}$ consist of $\mathfrak{Q}_{1}$ and the totally unordered subsets of $Y$. Then $\mathfrak{T}_{I}=\mathfrak{T}\left(\mathbb{Q}_{0}\right), \mathfrak{T}_{E}=$ $\left.\mathcal{T}^{2} \mathfrak{Q}_{1}\right)$ and $\mathfrak{T}_{C}=\mathfrak{T}\left(\mathbb{Q}_{2}\right)$. The first equality is trivial, and the second equality is a consequence of the main theorem in [10]. The last equality is nontrivial and requires several technical lemmas regarding the $\mathfrak{T}_{C}$-closed sets in $Y$.

If $(X, \mathcal{P}, \leq)$ is a semitree, then $(X, \leq)$ is Dedekind complete [21], and the concept of Dedekind closed as defined by Wolk [21] is exactly the same thing as our concept of closed given above. However, Wolk's definition was made in an arbitrary partially ordered set.

Lemma 2.25. If $\varnothing \neq A \subset X$ is chainable and closed, then $A$ is closed above and below.

Proof. Suppose that $A$ is chainable and closed, and let $a_{0}=\inf A$. If $a \in A$, then $A \cap\left[a_{0}, a\right]=A^{\prime} \neq \varnothing$. We claim that $a_{0}=\inf A^{\prime}$. To prove the claim, set $a^{\prime}=$ inf $A^{\prime}$ and observe that $a_{0} \leq a^{\prime}$. Also $a^{\prime} \in A$ since $A$ is closed. We prove that $a^{\prime}$ is a lower bound for $A$. Let $b \in A$. By Lemma 2.19 (i) and the fact that $A$ is chainable, $a^{\prime} \wedge b \in A$. But $a_{0} \leq a^{\prime} \wedge b \leq a^{\prime} \leq a$ since $a_{0} \leq a^{\prime}, b$. Thus $a^{\prime} \wedge b \in A^{\prime}$, and as a consequence $a^{\prime} \leq a^{\prime} \wedge b \leq b$. Since $a_{0}=\inf A, a_{0}=a^{\prime} \in A$. Thus $A$ is closed below. Next let $a_{0}$ be a quasi-least upper bound of $A$. Then $A \cap\left[e, a_{0}\right] \neq$ $\varnothing$; otherwise there would be an $x, e<x<a_{0}$, such that $x \not a$ for all $a \in A$. Further, $\sup A \cap\left[e, a_{0}\right]=a_{0}$ since $A$ is chainable, and hence, $a_{0} \in A$. Thus $A$ is closed above.

Lemma 2.26. If $A$ is a nonempty, chainable subset of $X$ which is closed above and below, then $A$ is closed.

Proof. Let $a_{0}=\inf A$. Then $a_{0} \in A$ since $A$ is closed below. Now let $A^{\prime}=$ 
$A \cap[x, y] \neq \varnothing$ where $x \leq y$, and let $a^{\prime}=\inf A^{\prime}$. Then there exists an $a \in A^{\prime}$ such that $a^{\prime} \leq a$ and so $a_{0}, a^{\prime} \in L(a)$. If $a^{\prime} \leq a_{0}$, then either $a^{\prime}=a_{0}$ or we contradict the fact that $a^{\prime}=\inf A^{\prime}$. Thus $a_{0} \leq a^{\prime} \leq a$ and so $a^{\prime} \in\left[a_{0}, a\right] \subset A$ since $A$ is chainable. Next let $b_{0}=\sup A^{\prime}$, and let $\left[e, b_{1}\right]$ be a ray which contains $\left[a_{0}, b_{0}\right]$. Now set

$$
b_{2}=\inf \left\{x \in L\left(b_{1}\right): a \leq x \text { for all } a \in A \cap L\left(b_{1}\right)\right\} \text {. }
$$

Then either there exists $a \in A$ such that $b_{2}<a$ or else $b_{2}$ is a quasi-least upper bound of $A$ and hence, $b_{2} \in A$. In either case $\left[a_{0}, b_{2}\right] \subset A$, and so $b_{0} \in A$ and $A$ is closed.

Corollary 2.27. If $A$ is nonempty and chainable, then $A$ is closed if and only if $A$ is closed above and below.

A partial order $\preceq$ on a topological space $(Y, \mathcal{T})$ is said to be semicontinuous if and only if $L(y)=\{z \in Y: z \preceq y\}$ and $M(y)=\{z \in Y: y \preceq z\}$ are $\mathcal{T}$-closed for each $y \in Y$. Evidently this is equivalent to requiring that $\mathfrak{T}$ contain the interval topology $\mathfrak{J}_{l}$ of O. Frink [4].

Lemma 2.28. The following bold:

(i) Botb $\varnothing$ and $X$ are closed.

(ii) If $x \leq y$, then $[x, y]$ is closed.

(iii) The partial order $\leq$ is semicontinuous.

(iv) The intersection of closed sets is closed.

(v) The union of a finite number of closed sets is closed.

(vi) Each $P \in \mathcal{P}$ is closed.

(vii) The set $X \backslash(M(x) \backslash\{x\})$ is chainable and closed for each $x \in X$.

Proof. Statement (i) follows immediately from the definition and Lemmas 2.22 and 2.23. Statement (ii) follows from Lemmas 2.22 and 2.23 and the fact that $[x, y]$ is totally ordered when $x \leq y$. For (iii) note that (ii) implies that $L(x)$ is closed for each $x$. Thus suppose that $A=M(x) \cap[y, z] \neq \varnothing$, where $y \leq z$. Then $x$ is a lower bound for $A$ and so inf $A \in M(x)$, and clearly $x \leq \sup A$. Hence, (iii) follows. Now let $\mathcal{C}$ be a collection of closed sets, and let $C=\cap \mathcal{C}$. Let $A=$ $C \cap[x, y] \neq \varnothing$ where $x \leq y$, and let $a_{0}=\inf A$ and $b_{0}=\sup A$. Then $A=C \cap$ $\left[a_{0}, b_{0}\right]$, and if $C^{\prime} \in \mathcal{C}$, then $A \subset C^{\prime} \cap\left[a_{0}, b_{0}\right]$ since $C \subset C^{\prime}$. Further, $a_{0} \leq \inf \left(C^{\prime} \cap\left[a_{0}, b_{0}\right]\right) \leq \inf A=a_{0}$ and $b_{0}=\sup A \leq \sup \left(C^{\prime} \cap\left[a_{0}, b_{0}\right]\right) \leq b_{0^{\circ}}$

Thus since $C^{\prime}$ is closed $a_{0}, b_{0} \in C^{\prime}$ for all $C^{\prime} \in \mathcal{C}$, and so $a_{0}, b_{0} \in C$ and $C$ is closed. For (v) let $A_{1}, A_{2}$ be closed and let $B=\left(A_{1} \cup A_{2}\right) \cap[x, y]$ where $x \leq y$ and $B \neq \varnothing$. Let $b_{0}=\inf B$ and $a_{i}=\inf \left(A_{i} \cap[x, y]\right), i=1,2$. (If $A_{i} \cap[x, y]=\varnothing$ 
for $i=1$ or 2 , we are done since then $B=A_{i} \cap[x, y]$ for $j \neq i$.) We claim that $b_{0}=a_{1}$ or $b_{0}=a_{2}$. For suppose $b_{0} \neq a_{1}$. Then $b_{0}<a_{1}$, and so $b_{0}=$ $\inf \left(\left[x, a_{1}\right] \cap A_{2}\right)=a_{2}$. Thus, since $A_{1}$ and $A_{2}$ are closed, $b_{0} \in A_{1}$ or $b_{0} \in A_{2}$. $A$ similar argument shows that $\sup B \in A_{1} \cup A_{2}$. Hence, $A_{1} \cup A_{2}$ is closed. Then ( $v$ ) follows by induction.

If $P \in \mathcal{P}$, then $P=[x, y]=[x \wedge y, x] \cup[x \wedge y, y]$, and so (vi) follows from (ii) and (v).

Finally, let $x \in X$ and suppose that $y, z \notin M(x) \backslash\{x\}$. Then

$$
([y \wedge z, y] \cup[y \wedge z, z]) \cap(M(x) \backslash\{x\})=\varnothing
$$

and $X \backslash(M(x) \backslash\{x\})$ is chainable. Next let

$$
A=(X \backslash(M(x) \backslash\{x\})) \cap[y, z] \neq \varnothing \quad \text { where } y \leq z .
$$

Then $x \nless \inf A$ and $x \nless \sup A$ since $a \in A$ implies that $x \nless$ a. Thus $X \backslash(M(x) \backslash\{x\})$ is closed.

The final lemma we need on the structure of the triple $(x, \mathcal{P}, \leq)$ is a very important separation property.

Lemma 2.29. If $A$ is chainable, and if $A$ meets both $M(x)$ and $X \backslash M(x)$, then $x \in A$.

Proof. Let $a_{1} \in A \cap M(x)$ and $a_{2} \in A \cap(X \backslash M(x))$. By Lemma 2.19,

$$
\left[a_{1}, a_{2}\right]=\left[a_{1} \wedge a_{2}, a_{1}\right] \cup\left[a_{1} \wedge a_{2}, a_{2}\right] \text {. }
$$

Further, $a_{1} \wedge a_{2}, x \in L\left(a_{1}\right)$ and since $a_{2} \notin M(x)$, it follows that $a_{1} \wedge a_{2} \leq x \leq a_{1}$, and so $x \in\left[a_{1} \wedge a_{2}, a_{1}\right]$. Thus $x \in\left[a_{1}, a_{2}\right] \subset A$.

3. The axioms on $\mathcal{F}$. In this section we give the three axioms needed on the family $\mathcal{F}$ and develop some properties resulting from these axioms. In the following we shall assume that Axioms 1-6 hold.

Axiom 7. If $x \leq y$, then $F([x, y])$ is chainable for all $F \in \mathcal{F}$.

Remark. It is easy to show that Axiom 7 is equivalent to: $F(P)$ is chainable for all $P \in \mathcal{P}$ and $F \in \mathcal{F}$. (This uses Axiom 4.) Also an immediate result of Axiom 7 is that $F(x)$ is chainable for each $x \in X$ and $F \in \mathcal{F}$. That is, each $F \in \mathcal{F}$ is point chainable.

Axiom 8. For all $F \in \mathcal{F}$ and for all $x \in X, F(x)$ is closed. That is, $F$ is point closed for all $F \in \mathcal{F}$.

Lemma 3.1. For all $F \in \mathcal{F}$ and for all $x \in X, F(x)$ is closed above and below.

Proof. By Axiom 8, $F(x)$ is closed, and hence, closed above and below by Lemma 2.25 for all $F \in \mathcal{F}, x \in X$. 
Lemma 3.2. For eacb $F \in \mathcal{F}$ and for all $x, y, z \in X$ sucb that $A=F(x) \cap$ $[y, z] \neq \varnothing$ we bave

(i) If $A \subset[y \wedge z, y]$, then inf $A=a_{1} \in F(x)$, sup $A=a_{2} \in F(x)$, and $A=$ $\left[a_{1}, a_{2}\right]$ is an ordered chain.

(ii) If $y$ and $z$ are not comparable, and if

$$
F(x) \cap(y \wedge z, y] \neq \varnothing \neq F(x) \cap(y \wedge z, z],
$$

then $y \wedge z=\inf A \in F(x)$. Furtber, the quasi-least upper bounds of $A$,

$$
s=\sup (F(x) \cap[y \wedge z, y]) \text { and } t=\sup (F(x) \cap[y \wedge z, z]) \text {, }
$$

are contained in $F(x)$. In this case $A=[s, t]$ is an unordered chain.

Proof. Both parts follow from the definition of chainable sets and closed sets.

Axiom 9. For all $F \in \mathcal{F}$ either (a) $F^{-1}(x)$ is chainable or (b) $F^{-1}(x)$ is closed for all $x \in X$.

Examples have been found which show that Axioms 1-9 are independent. Moreover, each of Axioms 1-9 is necessary in the sense that no one axiom can be omitted and still have $(X, \mathcal{P}, \mathcal{F})$ a fixed point structure under the remaining eight axioms [8].

We now prove a key lemma. This lemma uses Axioms 1-8 in its proof.

Lemma 3.3. If $F \in \mathcal{F}$ bas no fixed points, then (a) if $e \leq y<x$ implies tbat $F(y) \subset M(y)$, then $F(x) \subset M(x)$ and (b) there exists an $x_{0} \in X$ which is maximal with respect to $e \leq y \leq x_{0}$ implies $F(y) \subset M(y)$.

Proof. (a) Suppose that $F(x) \not \subset M(x)$. Then there is a $z \in F(x) \backslash M(x)$, and hence, $x \wedge z<x$. Thus $(x \wedge z, x) \neq \varnothing$. If $y \in(x \wedge z, x)$, then $F(y) \subset M(y)$ and so $F([y, x])$ meets both $M(y)$ and $X \backslash M(y)(z \in F(x)$ and $z \notin M(y)$ since $x \wedge z<y<x)$. Therefore $y \in F([y, x])$ by Axiom 7 and Lemma 2.29. But if $y^{\prime} \in[y, x)$, then $F\left(y^{\prime}\right) \subset M\left(y^{\prime}\right)$ and so $y \notin F\left(y^{\prime}\right)$. Thus $y \in F(x)$. This shows that $(x \wedge z, x) \subset F(x)$. But then Axiom 8 and Lemma 3.2 imply that $x=\sup (F(x) \cap[x \wedge z, x]) \in F(x)$, contrary to the assumption that $F$ has no fixed point. Hence, $F(x) \subset M(x)$.

(b) Let

$$
\mathcal{S}=\{[e, x]: e \leq y \leq x \text { implies } F(y) \subset M(y)\} .
$$

Since $F(e) \subset M(e)=X$ and since $\{e\}=[e, e], \delta$ is nonempty. Partially order $\delta$ by inclusion, and let $\pi$ be a nest in $\delta$. Then $U \pi$ is totally ordered in $X$, and hence, $\sup \cup \pi=x$ exists in $X$ by Lemma 2.23. Then $[e, x]$ is an upper bound for $\pi$ since $[e, x] \in \mathcal{S}$ follows from part (a). Hence, by Zorn's lemma, $\delta$ contains a maximal element. If $\left[e, x_{0}\right]$ is maximal in $\delta$, then $x_{0}$ is maximal in $X$ with respect to $e \leq y \leq x_{0}$ implies $F(y) \subset M(y)$. 
4. The main theorem. We are now ready to prove our main theorem. Before proceeding we shall reiterate our standing hypotheses. We are considering the triple $(X, \mathcal{P}, \mathcal{F})$ where $X$ is a nonempty set, $\mathcal{P}$ is a nonempty collection of subsets of $X$ and $\mathcal{F}$ is a nonempty family of multifunctions on $X$ into $X$. In addition we are assuming that Axioms $1-9$ hold. Then $(X, \mathcal{P}, \mathfrak{F})$ is a fixed point structure in case each $F \in \mathcal{F}$ has a fixed point.

Theorem 4.1. The triple $(X, \mathcal{P}, \mathcal{F})$ is a fixed point structure.

Proof. Suppose that there exists an $F \in \mathcal{F}$ which does not have a fixed point, and let $x_{0}$ be a maximal element of $X$ with respect to $e \leq y \leq x_{0}$ implies $F(y) \subset$ $M(y)$ ( $x_{0}$ exists by Lemma 3.3). Let $x_{1}=\inf F\left(x_{0}\right)$. By Lemma 3.2, $x_{1} \in F\left(x_{0}\right)$, and since $F\left(x_{0}\right) \subset M\left(x_{0}\right) \backslash\left\{x_{0}\right\}, x_{0}<x_{1}$. Choose $z_{1} \in\left(x_{0}, x_{1}\right)$.

(1) Claim. If $x_{0}<z<z_{1}$, then there is a $y \in\left(x_{0}, z\right)$ such that $z_{1} \in F(y)$; i.e. $\left(x_{0}, z\right) \cap F^{-1}\left(z_{1}\right) \neq \varnothing$. To establish the claim note that there is an $x \in\left(x_{0}, z\right)$ such that $F(x) \not \subset M(x)$ for otherwise the maximality of $x_{0}$ is contradicted. Further, $M\left(z_{1}\right) \subset M(x)$ and so $F\left(\left[x_{0}, x\right]\right)$ meets both $M\left(z_{1}\right)$ and its complement. Then by Lemma 2.29, there is a $y \in\left[x_{0}, x\right]$ such that $z_{1} \in F(y)$, and the claim is established since $z_{1} \in F\left(x_{0}\right)$ contradicts $z_{1}<x_{1}=\inf F\left(x_{0}\right)$.

We now have to consider two possibilities.

(2) The first of these is that $F^{-1}\left(z_{1}\right)$ is closed. But then by (1)

$$
x_{0}=\inf \left(\left[x_{0}, z_{1}\right] \cap F^{-1}\left(z_{1}\right)\right) \in F^{-1}\left(z_{1}\right) \text {. }
$$

That is $z_{1} \in F\left(x_{0}\right)$, which is a contradiction.

(3) Next suppose that $F^{-1}\left(z_{1}\right)$ is chainable. Then (1) shows that there is a $y \in\left(x_{0}, z_{1}\right)$ such that $\left(x_{0}, y\right] \subset F^{-1}\left(z_{1}\right)$. That is, for all $x, x_{0}<x \leq y, z_{1} \in F(x)$ which implies that $F(x) \cap M(x) \neq \varnothing$. But then $F(x) \subset M(x)$ since by assumption $x \notin F(x)$ for all $x \in X$. Thus $[e, y] \in \mathcal{S}$ (see the proof of Lemma 3.3(b)) and $x_{0}<y$. This is also a contradiction.

In both of the above cases, we were led to a contradiction. Thus each $F \in \mathcal{F}$ must have a fixed point.

5. Some fixed point structures and applications. In this section we describe three fixed point structures whose origins lie in three quite different mathematical structures. In each case we derive, as applications, fixed point theorems some of which are new and some of which are known theorems.

Example 1. Let $X$ be an arcwise connected topological space in which every nest of arcs is contained in an arc. (In this example an arc is a compact, connected, Hausdorff space with exactly two noncutpoints called the endpoints, and an arc need not be separable.) Then set $\mathcal{P}=\{A \subset X: A$ is an arc $\}$. (Here $\{x\}$ is considered to be an arc with endpoints $x, x_{0}$ ) First observe that $X$ is acyclic [7] and in fact 
$X$ has unique arcs. Then it is easy to see that Axioms $1,3,4,5$ and 6 are satisfied. For example, Axiom 5 is implied by the hypothesis that each nest of arcs is contained in an arc. For Axiom 2 let $\mathscr{P}_{0} \subset \mathcal{P}$ and let $P_{0} \in \mathscr{P}_{0}$. Set $\mathscr{P}_{1}=\left\{P_{0} \cap P\right.$ : $\left.P \in \mathscr{P}_{0}\right\}$. Then $\cap \mathscr{P}_{0}=\cap \mathscr{P}_{1}$, but $\cap \mathscr{P}_{1}$ is the intersection of arcs in an arc and so is either empty or an arc.

Now let $\mathcal{F}$ be the collection of point closed multifunctions on $X$ into $X$ such that if $F \in \mathcal{F}$, then $F(P)$ is arcwise connected for each $P \in \mathcal{P}$ and $F^{-1}(x)$ is either chainable or closed for each $x \in X$. That is, $\mathcal{F}$ is a family of multifunctions which satisfies Axioms 7, 8 and 9. (Observe that the topology on $X$ defined in $\$ 2$ is larger than the given topology on $X$.) Note that by a result of J. K. Harris [5], $\mathcal{F}$ contains the single-valued, continuous functions. It now follows that Axioms 1-9 are consistent. Then $(X, \mathscr{P}, \mathcal{F})$ is a fixed point structure by Theorem 4.1.

We now obtain a number of corollaries to this result. $F$ irst, the theorem of Wallace [16]:

Corollary 5.1. Eacb u.s.c., continuum-valued multifunction on a tree into itself bas a fixed point.

We also get Ward's [20] generalization of Wallace's theorem. The hypothesis of hereditary decomposability in Ward's theorem [20] was recently shown to be redundant by David P. Bellamy [2] who proved that an arcwise connected, Hausdorff continuum is decomposable. Results of Harris [5] and Ward [19] then apply to yield the following corollary.

Corollary 5.2. If $X$ is an arcwise connected, bereditarily unicoberent, Hausdorff continuum, then every u.s.c., continuum-valued multifunction $F: X \rightarrow X$ bas a fixed point.

Next we obtain a theorem of Smithson [9].

Corollary 5.3. If $X$ is a tree, then each point closed, connected, monotone multifunction on $X$ into $X$ bas a fixed point.

We also have the following new result.

Corollary 5.4. Let $X$ be an arcwise connected, bereditarily unicoberent, Hausdorff continuum. If $F: X \rightarrow X$ preserves continua and if $F^{-1}(x)$ is either closed or arcwise connected for each $x \in X$, then $F$ bas a fixed point.

Finally, we have Smithson's [12] generalization of a theorem of Young-Mohler [22], [7].

Corollary 5.5. Let $X$ be an arcwise connected space in which every nest of arcs is contained in an arc. If $F: X \rightarrow X$ is point closed, maps arcs onto arcwise connected sets, and if $F^{-1}(x)$ is either closed or arcwise connected for each $x \in X$, then $F$ bas a fixed point. 
Example 2. Let $(X, \leq)$ be a partially ordered set such that:

(a) There is a least element $e \in X$.

(b) The partial order $\leq$ is order dense.

(c) If $\varnothing \neq A \subset X$, then inf $A$ exists in $X$.

(d) Each nonempty, totally ordered subset of $X$ has a supremum in $X$.

(e) For each $x \in X, L(x)$ is totally ordered.

We state (a) merely to be explicit; in fact, (c) implies (a). Now suppose that $a \leq b$, set $[a, b]=\{x \in X: a \leq x \leq b\}$. Then from (e) we get

Lemma 5.6. If $a \leq b$, then the set $[a, b]$ is totally ordered.

Then from Lemma 5.6 we get

Lemma 5.7. If $a \leq x \leq b$, then $[a, b]=[a, x] \cup[x, b]$.

Now if $a$ and $b$ are not comparable, then set $[a, b]=[a \wedge b, a] \cup[a \wedge b, b]$. Let $\mathcal{P}$ be the collection of all sets $[a, b]$ for $a, b \in X$.

Now Axioms 1 and 3 are immediate, and Axiom 5 follows from (d) and Lemma 5.6. Axiom 2 follows from Lemmas 5.6 and 5.7 by using the same technique as in Example 1. Axiom 4 also follows from Lemmas 5.6 and 5.7 and Axiom 6 is (b). (Conversely, observe that any semitree $(X, \mathcal{P}, \leq)$ satisfies (a)-(e).)

Since $(X, \mathcal{P})$ satisfies Axioms 1-6, we have the notion of closed set as defined in $\$ 2$. Thus let $\mathcal{F}$ be the set of multifunctions on $X$ into $X$ which satisfy Axioms 7,8 and 9 . Then $(X, \mathcal{P}, \mathcal{F})$ is a fixed point structure by Theorem 4.1 . We now state a new fixed point theorem for partially ordered sets.

Theorem 5.8. Let $(X, \leq)$ be a partially ordered set whicb satisfies conditions (a)-(e). Let $F: X \rightarrow X$ be a multifunction such that $F([a, b])$ and $F^{-1}(x)$ are chainable for all $a, b, x \in X$. If, in addition, the quasi-least upper bounds and the infimum of $F(x)$ are contained in $F(x)$ for all $x \in X$, then $F$ bas a fixed point.

In Theorem 5.8, the sets $[a, b]$ are as defined above, and the terms chainable and quasi-least upper bound are as used before.

Remark. Suppose that $f: X \rightarrow X$ is order preserving. Then $f^{-1}(x)$ need not be chainable or closed. Hence, we cannot derive a theorem regarding isotone maps from Theorem 4.1. However, from previous work on isotone maps, a theorem analogous to Theorem 4.1 can be obtained by replacing Axioms 7-9 by an axiom requiring that each function be isotone or more generally by the two conditions in [11]. But then we do not need the acyclicity or order density inherent in Axioms 1-6, and so some modifications of these are indicated. This will be done in $\$ 6$ where we will get Tarski's theorem [15] as a corollary.

Example 3. Let $V$ be a real vector space, and let $e \in V$. A line $L$ through $e$ is a set $L=e+U$ where $U$ is a one dimensional linear subspace of $V$. A closed 
line segment is a set

$$
\left[x_{1}, x_{2}\right]=\left\{x \in V: x=\lambda x_{1}+(1-\lambda) x_{2}, 0 \leq \lambda \leq 1\right\} .
$$

A subset $X$ of $V$ is star-shaped relative to $e \in X$ in case $[e, x] \subset X$ for all $x \in X$, and $X$ is a closed star at $e$ in case $e \in X$ and each line $L$ through $e$ intersects $X$ in a closed line segment.

Observe that if $X$ is a closed star at $e$, then $X$ is star-shaped relative to $e$.

Now let $X$ be a closed star at $e$ and let $x, y \in X$. Redefine the symbol $[x, y]$ as follows: If $x, y$ are not in a line through $e$, then $[x, y]=[e, x] \cup[e, y]$ where $[e, x]$ and $[e, y]$ are the closed line segments as defined above. If $x, y$ are in a line through $e$, then $[x, y]$ is the line segment from $x$ to $y$. Now $\mathcal{P}=\{[x, y]: x$, $y \in X\}$.

It is easy to prove Lemmas 2.7, 2.13 and 2.17 directly in the present setting by using the vector space structure. Axioms 1 and 3 follow from the definition of $\mathcal{P}$, and Axiom 6 follows from the definition of line segments. Axiom 2 is obtained by observing that a member of $\mathcal{P}$ is either a line segment or the union of two line segments with a common endpoint, and the intersection of such sets is one of these kinds of sets. Axiom 5 follows by first considering nests of segments with one endpoint $e$ and then extending the argument to the general case. Finally, Axiom 4 is verified by a routine consideration of the possible cases.

Next let $\mathcal{F}$ be the set of multifunctions on $X$ into $X$ which satisfy Axioms 7,8 and 9 . Then $(X, \mathscr{P}, \mathcal{F})$ is a fixed point structure by Theorem 4.1. We restate this in the following two corollaries. In these we use the term order closed to mean closed in the sense of $\$ 2$.

Corollary 5.9. Let $X$ be a closed star at $e$. If $F([x, y])$ is chainable for each $[x, y] \in \mathcal{P}$, if $F(x)$ is order closed for all $x \in X$ and if $F^{-1}(x)$ is either chainable or order closed for all $x \in X$, then $F$ bas a fixed point.

Corollary 5.10. Let $X$ be a compact, convex subset of a Hausdorff, real topological vector space, and let $e \in X$. Then $X$ is a closed star at $e$, and if $F: X \rightarrow X$ satisfies the bypothesis of Corollary 5.9 (chains defined relative to e), then $F$ has a fixed point.

Remark. A closed star in a topological vector space need not be either topologically closed or compact, and the conditions placed on the multifunctions do not imply continuity. In the case of a one dimensional line segment, we get the Kakutani [6] (and Brouwer) theorem by taking $e$ to be one of the endpoints. Unfortunately, this does not work in two (or higher) dimensional spaces. In one sense Corollary 5.10 is more general than the Kakutani theorem, because the multifunction need not be continuous. However, the problem of obtaining the Kakutani theorem and its generalizations as a fixed point structure is still unsolved. 
It should also be possible to generalize Corollary 5.9 to a set $X$ that is the union of a family of closed stars suitably linked by closed line segments in much the way a tree is constructed. But we will not do this.

6. Some altemate fixed point structures. In this section we obtain two alternate fixed point structures by using some or all of Axioms 1-6 with replacements for Axioms 7-9. We also present several applications of these structures.

For the first different structure, let $(X, \mathscr{P})$ satisfy Axioms $1-6$ and let $\leq$ be the partial order with least element $e$ as defined in $\$ 2$. Recall that closed sets were also defined in $\$ 2$ and that Lemma 2.28 showed that the collection of closed sets formed the closed sets of a topology which we called the chain topology and denoted by $\mathfrak{T}_{C}$. We now give two alternate axioms on the collection $\mathcal{F}$ of multifunctions.

Axiom 10. For each $F \in \mathcal{F}$ and each $x \in X, F(x)$ is chainable.

Axiom 11. Each $F \in \mathcal{F}$ is l.s.c. with respect to the chain topology $\mathfrak{T}_{C}$.

Now assume that $(X, \mathcal{P}, \mathcal{F})$ satisfies Axioms $1-6$ and Axioms $10-11$. We shall need the following lemma which parallels Lemma 3.3 in the present setting.

Lemma 6.1. Suppose that $F \in \mathcal{F}$. If $e \leq y<x$ implies that $F(y) \subset M(y)$, then $F(x) \subset M(x)$. Further, there exists an $x_{0} \in X$ which is maximal with respect to $y \in L\left(x_{0}\right)$ implies $F(y) \subset M(y)$.

Proof. Suppose there exists $z \in F(x) \backslash M(x)$. Then $x \wedge z=z^{\prime}<x$. Thus choose $t \in\left(z^{\prime}, x\right)$, and note that $t \not z$. Therefore $z \in(X \backslash M(t)) \cap F(x)$, and so, since $F$ is 1.s.c., there is an open set $V, x \in V$, such that $F(y) \cap(X \backslash M(t)) \neq \varnothing$ for all $y \in V$. Now set $U=(M(t) \backslash\{t\}) \cap V$. (Note that $M(t) \backslash\{t\}$ is open by Lemma 2.28 (vii).) Further, the definition of $\mathfrak{T}_{C}$ implies that there exists $y \in U$ such that $y<x$. Then $F(y) \subset M(y)$ by hypothesis, but $F(y) \cap(X \backslash M(t)) \neq \varnothing$ (from the construction of $U$ ) which is a contradiction since $M(y) \subset M(t)$. Thus $F(x) \subset M(x)$.

To prove the last statement, use the Hausdorff Maximality Principle (or Zorn's lemma) and the first statement.

Theorem 6.2. The triple $(X, \mathcal{P}, \mathfrak{F})$ is a fixed point structure.

Proof. Let $F \in \mathcal{F}$ and let $x_{0} \in X$ be maximal with respect to $e \leq y \leq x_{0}$ implies $F(y) \subset M(y)$. Now let $z \in F\left(x_{0}\right)$ and let $t \in\left(x_{0}, z\right)$. (If $z=x_{0}$, we are done.) Since $F$ is 1.s.c., there exists an open set $V$ such that $x_{0} \in V$ and $F(x)$ $\cap(M(t) \backslash\{t\}) \neq \varnothing$ for all $x \in V$. Now from the definition of $\mathcal{T}_{C}$, we obtain a $t^{\prime} \in V$ such that $x_{0}<t^{\prime} \leq t$ and $\left[x_{0}, t^{\prime}\right] \subset V$. Then by the maximality of $x_{0}$, there exists a $y \in\left(x_{0}, t^{\prime}\right)$ such that $F(y) \not \subset M(y)$. But $y \in V$; hence, $F(y) \cap M(t) \neq \varnothing$ and $M(t)$ $C M(y)$. Thus $F(y)$ meets both $M(y)$ and its complement. Finally, since $F(y)$ is chainable, $y \in F(y)$ by Lemma 2.29 , and we have a fixed point.

A corollary of the proof of Theorem 6.2 which proves to be useful is the following: 
Corollary 6.3. Let $\mathcal{T}$ be another topology for $X$ sucb that $X \backslash M(t)$ and $M(t) \backslash\{t\}$ are $\mathcal{T}$ open sets for each $t \in X$. If $F:\left(X, \mathfrak{T}_{C}\right) \rightarrow(X, \mathfrak{T})$ is l.s.c. and point chainable, then $F$ bas a fixed point.

Now if $(X, \mathfrak{T})$ is a tree, then $(X, \mathscr{P})$ satisfies Axioms $1-6$ where $\mathscr{P}$ is the set of arcs in $X$. (This follows from Ward's characterization of a tree [17].) Further, it can be shown that $\mathscr{T} \subset \mathcal{I}_{C}$, and hence, the identity map $i:\left(X, \mathscr{T}_{C}\right) \rightarrow$ $(X, \mathcal{I})$ is continuous. Thus if $F:(X, \mathcal{T}) \rightarrow(X, \mathcal{I})$ is l.s.c. and point chainable, so is $F \circ i:\left(X, \mathfrak{I}_{C}\right) \rightarrow(X, \mathcal{J})$. Thus by Corollary 6.3 we get the following theorem of Smithson [12].

Corollary 6.4. If $X$ is a tree and if $F: X \rightarrow X$ is a l.s.c. multifunction sucb that $F(x)$ is arcwise connected for each $x \in X$, then $F$ bas a fixed point.

Finally, we get the following new result as a corollary of Theorem 6.2.

Corollary 6.5. Let $(X, \mathcal{I})$ be an arcwise connected space in which each nest of arcs is contained in an arc. If $\mathfrak{T}=\mathfrak{T}_{C}$, if $F: X \rightarrow X$ is l.s.c. and if $F(x)$ is arcwise connected for each $x \in X$, then $F$ bas a fixed point.

For the next fixed point structure, we shall assume that $(X, \leq)$ is a partially ordered set with least element $e$, and we shall use a modification of Axiom 5 together with two new axioms on the set of multifunctions $\mathcal{F}$.

Axiom 5s. Each nonempty, totally ordered subset of $X$ has a supremum in $X$.

Observe that Axiom 5s is merely condition (d) in Example 2 of $\$ 5$. Moreover, Axiom $5 s$ is actually equivalent to Axiom 5 if one assumes the remaining five axioms defining a semitree.

Axiom 12. Let $x_{1} \leq x_{2} \in X$, and let $F \in \mathcal{F}$. If $y_{1} \in F\left(x_{1}\right)$, then there exists a $y_{2} \in F\left(x_{2}\right)$ such that $y_{1} \leq y_{2}$.

Axiom 13. Let $F \in \mathcal{F}$ and let $C$ be a totally ordered subset of $X$. Suppose there is an isotone single-valued function $g: C \rightarrow X$ such that $g(x) \in F(x)$ for all $x \in C$. If $x_{0}=\sup C$, then there exists $y_{0} \in F\left(x_{0}\right)$ such that $g(x) \leq y_{0}$ for all $x \in C$.

We interpret Axioms 12-13 to mean that the given conditions hold for all $F \in \mathcal{F}$. Note that every isotone single-valued function satisfies both Axioms 12 and 13.

Now let $(X, \mathcal{F})$ satisfy Axioms 5s, 12, and 13. Then by Theorem 1.1 in [11] (which generalizes a theorem of Abian and Brown [1]), we have

Theorem 6.6. Every $F \in \mathcal{F}$ bas a fixed point.

Now Theorem 6.6 is nothing but a variant of Theorem 1.1 in [11]. Furthermore, the chains in $X$ do not explicitly appear in Theorem 6.6. For these reasons, we now wish to give a modification of the above structure on $X$ that is more in line with the semitree structure developed in $\$ 2$ of this paper. This modification will none the less have fixed point theorems of Ward [18] and Tarski [15] as corollaries. 
Explicitly, we assume that $(X, \mathcal{P})$ is subject to Axiom 3 and the following modifications of Axioms 1,2 and 5 where $e$ is some fixed point of $X$.

Axiom $1^{\prime}$. For each $x, y \in X$, there is a unique minimal element $[x, y]$ of $\mathcal{P}$ containing $x$ and $y$. (See Lemma 2.1.)

Axiom 2'. If $\mathscr{P}_{0}=\left\{\left[e, b_{a}\right]: a \in \Gamma\right\} \subset \mathcal{P}$, then there is a $b \in X$ such that $\cap \mathcal{P}_{0}=[e, b]$.

Axiom $5^{\prime}$. If $\mathscr{P}_{0}=\left\{\left[e, b_{a}\right]: \alpha \in \Gamma\right\} \subset \mathcal{P}$ is a nest, then there is a $b \in X$ such that $\cup \mathcal{P}_{0} \subset[e, b]$.

Remarks. Axioms 5 and $5^{\prime}$ are equivalent when one assumes the remaining five axioms defining a semitree. Also Axiom 1 and Axiom $1^{\prime}$ are equivalent and Axiom 2 implies Axiom $2^{\prime}$ under similar assumptions as we have seen in $\$ 2$. Now Axioms $1^{\prime}, 2^{\prime}$ and 3 are all that are necessary to define the partial order $\leq$ with least element $e$ in $\$ 2$. However, an example has been constructed which satisfies Axioms $1,2,3$ and 5 (or $5^{\prime}$ ), but not $2^{\prime}$ or 4 , and this example contains a totally ordered subset with no supremum. Furthermore, a simple modification of this example shows that Axioms 1,2, 2', 3 and 5 do not in themselves imply that totally ordered sets have supremums. This is the reason for introducing Axiom $5^{\prime}$ as well as Axiom 2'.

Now assume that $(X, \mathcal{P}, \mathcal{F})$ satisfies Axioms $1^{\prime}, 2^{\prime}, 3,5^{\prime}, 12$ and 13. Further, assume that $\leq$ is the partial order with least element $e$ as defined in $\$ 2$. We now have the required property.

Lemma 6.7. If $\varnothing \neq A \subset X$ is totally ordered, then $A$ bas a supremum in $X$.

Proof. Let $\mathscr{P}_{0}=\{[e, a]: a \in A\}$. Then, by Axiom 5', there is a $b \in X$ such that $A \subset \cup \mathcal{P}_{0} \subset[e, b]$. Thus let $\mathcal{S}=\{[e, z]: A \subset[e, z]\}$. Then, by Axiom 2', there is an $x_{0} \in X$ such that $\bigcap \mathcal{S}=\left[e, x_{0}\right]$, and $x_{0}=\sup A$. (See the proof of Lemma 2.23.)

The following theorem is then a corollary of Lemma 6.7 and Theorem 6.6. We could of course prove this theorem directly without dependence on Theorem 6.6.

Theorem 6.8. The triple $(X, \mathcal{P}, \mathcal{F})$ is a fixed point structure.

Example. Let $(X, \leq)$ be a partially ordered set which is a complete semilattice with least element $e$ and in which each totally ordered set has a supremum. We define the elements of $\mathcal{P}$ as in Example 2 of $\$ 5$. Routine calculations show that Axioms $1^{\prime}, 2^{\prime}, 3$ and $5^{\prime}$ are satisfied. In fact, the converse is true; namely, if $(X, \mathcal{P})$ satisfies Axioms $1^{\prime}, 2^{\prime}, 3$ and $5^{\prime}$, then there is a partial order $\leq$ such that $(X, \leq)$ is a complete semilattice and each totally ordered set has a supremum (Lemma 6.7). But observe that $(X, \leq)$ need not satisfy Axioms 2, 4, 5 or 6 in any event, so that one sees how semitrees and complete lattices fit into the present scheme. Now let $\mathcal{F}$ be the class of multifunctions defined by Axioms 12 and 13 . Then, by Theorem 6.8 , each $F \in \mathcal{F}$ has a fixed point. Three corollaries are immediate. 
Corollary 6.9. (Ward [18].) Let $(X, \leq)$ be a semilattice and let $f: X \rightarrow X$ be an isotone function. If $X$ is compact in the interval topology [4] generated by taking all of the sets $L(x)$ and $M(x), x \in X$, as a subbas is for the closed sets, then $f$ bas a fixed point.

Proof. Since $X$ is compact in the interval topology and $L(x)$ is closed for each $x \in X, L(x)$ is compact for each $x \in X$. Thus by Theorem 1 of $[18],(X, \leq)$ is a complete semilattice. Then Axiom 5s follows by a standard compactness argument. So we apply the above example.

Corollary 6.10. (Tarski [15].) If $f: X \rightarrow X$ is an isotone function on a complete lattice into itself, then $f$ has a fixed point.

Corollary 6.11. If $(X, \mathcal{P}, \leq)$ is a semitree or if $(X, \leq)$ is a complete lattice, and if $F$ is a multifunction on $X$ into itself satisfying Axioms 12 and 13, then $F$ bas a fixed point.

The fixed point structures of Theorems 6.6 and 6.8 can be characterized in semilattices.

Theorem 6.12. Let $(X, \leq)$ be a semilattice with least element $e$ and let $\mathcal{P}, \mathcal{F}$ be defined as before. Then the following are equivalent:

(i) The triple $(X, \mathcal{P}, \mathcal{F})$ is a fixed point structure.

(ii) Every isotone function $f: X \rightarrow X$ bas a fixed point.

(iii) Every nonempty, totally ordered subset of $X$ bas a supremum in $X$; i.e. the pair $(X, \leq)$ satisfies Axiom $5 \mathrm{~s}$.

Proof. The missing step is (ii) implies (iii), and this was proved by Smithson [13].

In a complete semilattice, two additional necessary and sufficient conditions are available.

Theorem 6.13. Let $(X, \leq)$ be a complete semilattice with least element $e$ and let $\mathcal{P}, \mathcal{F}$ be defined as before. Then the following are equivalent:

(i) The triple $(X, \mathcal{P}, \mathcal{F})$ is a fixed point structure.

(ii) Every isotone function $f: X \rightarrow X$ bas a fixed point.

(iii) $X$ is compact in the interval topology.

(iv) The pair $(X, \mathcal{P})$ satisfies Axiom $5^{\prime}$.

(v) The pair $(X, \leq)$ satisfies Axiom 5 s.

Proof. Obviously (i) implies (ii) since $f \in \mathcal{F}$. Ward's proof [17] that (ii) implies (iii) is sufficient in the present setting. Conditions (iv) and (v) were shown to be equivalent in the last example, and (iii) implies ( $v$ ) was shown in the proof of Corollary 6.9. Finally, Smithson [11] proved that (v) implies (i) (Theorem 6.6).

Remarks. Ward [18] stated the equivalence of (ii) and (iii) in an arbitrary semilattice. An example shows that completeness is necessary in Theorem 6.13. Let 
$X=[0,1] \cup\{a, b\}$ with the usual ordering on $[0,1]$ and with $0<a, b<x$ for all $x \in(0,1]$. Then $(X, \leq)$ is a noncomplete semilattice with least element 0 satisfying Axiom 5s. Therefore (i) and (ii) hold, but $(X, \leq)$ is not compact in the interval topology since, for example, $M(a) \cap M(b)$ is not compact in the interval topology. If we turn $(X, \leq)$ upside down (i.e. consider $(X, \geq))$, we obtain an example showing the necessity of the semilattice structure in Theorem 6.12 .

\section{REFERENCES}

1. S. Abian and A. B. Brown, A theorem on partially ordered sets, with applications to fixed point theorems, Canad. J. Math. 13 (1961), 78-82. MR 23 \#A817.

2. David P. Bellamy, Composants of Hausdorff indecomposable continua, Pacific J. Math. (to appear).

3. C. E. Capel and W. L. Strother, Multi-valued functions and partial order, Portugal. Math. 17 (1958), 41-47. MR 21 \#322.

4. Orrin Frink, Jr., Topology in lattices, Trans. Amer. Math. Soc. 51 (1942), 569-582. MR 3, 313.

5. J. K. Harris, Order structures for certain acyclic topological spaces, Thesis, University of Oregon, Eugene, Ore., 1962.

6. S. Kakutani, A generalization of Brouwer's fixed point theorem, Duke Math. J. 8 (1941), 457-459. MR 3, 60.

7. Lee K. Mohler, $A$ fixed point theorem for continua which are hereditarily divisible by points, Fund. Math. 67 (1970), 345-358. MR 41 \#6196.

8. T. B. Muenzenberger, Fixed point structures, Thesis, University of Wyoming, Laramie, Wy., 1972.

9. R. E. Smithson, A fixed point theorem for connected multi-valued functions, Amer. Math. Monthly 73 (1966), 351-355. MR 33 \#1848.

10. - Topologies generated by relations, Bull. Austral. Math. Soc. 1 (1969), 297-306. MR $41 \# 2605$.

11. - Fixed points of order preserving multifunctions, Proc. Amer. Math. Soc. 28 (1971), 304-310. MR 43 \#114.

12. - Fixed point theorems for certain classes of multifunctions, Proc. Amer. Math. Soc. 31 (1972), 595-600. MR 44 \#5946.

13. - Fixed points in partially ordered sets, Pacitic J. Math. 45 (1973), 363-367.

14. Lynn A. Steen and J. Arthur Seebach, Jr., Counterexamples in topology, Holt, Rinehart and Winston, New York, 1970. MR $42 \# 1040$.

15. Alfred Tarski, $A$ lattice-theoretical fixpoint theorem and its applications, Pacific J. Math. 5 (1955), 285-309. MR 17, 574.

16. A. D. Wallace, A fixed point theorem for trees, Bull. Amer. Math. Soc. 47 (1941), 757-760. MR 3, 57.

17. L. E. Ward, A note on dendrites and trees, Proc. Amer. Math. Soc. 5 (1954), 992994. MR 17, 180.

18. - Completeness in semi-lattices, Canad. J. Math. 9 (1957), 578-582. MR 19, 938.

19. - A fixed point theorem for multi-valued functions, Pacific J. Math. 8 (1958), 921-927. MR 21 \#2215. 
20. L. E. Ward, Characterization of the fixed point property for a class of set-valued mappings, Fund. Math. 50 (1961/62), 159-164. MR 24 \#A2956.

21. E. S. Wolk, Ordercompatible topologies on a partially ordered set, Proc. Amer. Math. Soc. 9 (1958), 524-529. MR 20 \#3079.

22. Gail S. Young, Jr., The introduction of local connectivity by change of topology, Amer. J. Math. 68 (1946), 479-494. MR 8, 49.

DEPARTMENT OF MATHEMATICS, UNIVERSITY OF WYOMING, LARAMIE, WYOMING 82071

Current address (T. B. Muenzenberger): Department of Mathematics, Kansas State University, Manhattan, Kansas 66506

Current address (R. E. Smithson): Department of Mathematics, University of Houston, Houston, Texas 77004 